\title{
El aborto eugenésico podría explicar la menor mortalidad infantil existente en Cuba comparada con la de Chile
}

\author{
ENRIQUE DONOSO S. ${ }^{1}$, JORGE A. CARVAJAL C. ${ }^{1}$, PHD.
}

\section{Eugenic abortion could explain the lower infant mortality in Cuba compared to that in Chile}

Background: Cuba and Chile have the lower infant mortality rates of Latin America. Infant mortality rate in Cuba is similar to that of developed countries. Chilean infant mortality rate is slightly higher than that of Cuba. Aim: To investigate if the lower infant mortality rate in Cuba, compared to Chile, could be explained by eugenic abortion, considering that abortion is legal in Cuba but not in Chile. Material and Methods: We compared total and congenital abnormalities related infant mortality in Cuba and Chile during 2008, based on vital statistics of both countries. Results: In 2008, infant mortality rates in Chile were significantly higher than those of Cuba (7.8 vs. 4.7 per 1,000 live born respectively, odds ratio (OR) 1.67; 95\% confidence intervals (CI) 1.52-1.83). Congenital abnormalities accounted for 33.8 and $19.2 \%$ of infant deaths in Chile and Cuba, respectively. Discarding infant deaths related to congenital abnormalities, infant mortality rate continued to be higher in Chile than in Cuba (5.19 vs. 3.82 per 1000 live born respectively, OR 1.36; 95\%CI 1.221.52). Conclusions: Considering that antenatal diagnosis is widely available in both countries, but abortion is legal in Cuba but not in Chile, we conclude that eugenic abortion may partially explain the lower infant mortality rate observed in Cuba compared to that observed in Chile.

(Rev Med Chile 2012; 140: 999-1005).

Key words: Abortion legal; Abortion eugenic; Congenital abnormalities; Cuba; Chile; Infant mortality; Public Health.

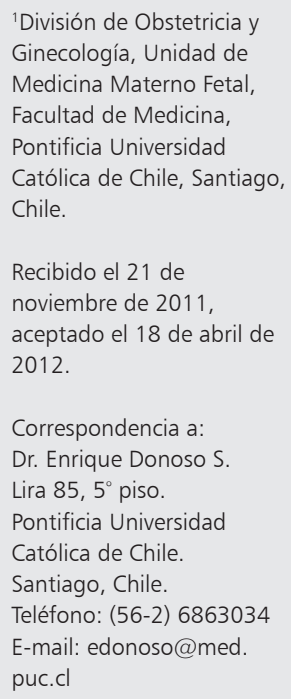

L a tasa de mortalidad infantil es un indicador del desarrollo económico, social y sanitario de un país. En los países menos desarrollados, la muerte del niño antes del primer año de vida es un dramático y no resuelto problema de salud pública ${ }^{1}$. Cuba y Chile, son parte de los países con menor mortalidad infantil en la Región de las Américas, alcanzando Cuba tasas de países desarrollados, manteniéndose Chile muy cerca de ellos².

En Cuba y Chile las causas de muerte en los primeros 28 días de vida dependen principalmente de malformaciones congénitas y prematurez, posteriormente, hasta alcanzar el primer año de vida, adquieren relevancia las enfermedades originadas de causas ambientales, especialmente las enfermedades infecciosas ${ }^{3,4}$. La reducción de la tasa de mortalidad infantil es un objetivo sanitario mundial, el que se ha logrado en las últimas décadas mejorando la atención de los recién nacidos prematuros y de los infantes con patologías infecciosas.

Las malformaciones congénitas no pueden ser reducidas mediante estrategias sanitarias, excepto por la suplementación prenatal con ácido fólico que reduce significativamente el riesgo de malformaciones del tubo neural. Así, las malformaciones congénitas aumentan su preponderancia como 
causa de muerte infantil, llegando a ser la primera causa en países desarrollados.

Gran parte de las malformaciones congénitas incompatibles con la vida extrauterina, como la anencefalia, la agenesia renal bilateral, las malformaciones multisistémicas y algunas trisomías, son identificables o sospechables mediante la ultrasonografía prenatal de rutina ${ }^{5-7}$. Frecuentemente los portadores de estas patologías nacen vivos y fallecen en el período neonatal precoz, incrementando la mortalidad infantil. Es posible que el aborto eugenésico, permisible en múltiples legislaciones, pueda reducir la mortalidad infantil, al evitar el nacimiento de niños malformados o no viables.

No ha sido estudiado de modo específico si la interrupción legal del embarazo por malformaciones congénitas tiene un impacto demográfico en la tendencia de la mortalidad infantil, sin embargo, algunas experiencias internacionales así lo sugieren ${ }^{8-12}$. En Canadá, la tasa de mortalidad infantil atribuible a malformaciones congénitas se ha reducido en los últimos 20 años, muy probablemente debido a las políticas de diagnóstico antenatal y aborto eugenésico ${ }^{8-10}$.

Cuba y Chile tienen una legalidad contrapuesta en relación a la interrupción de la gestación antes de la viabilidad: Cuba tiene una legislación que no penaliza el aborto inducido ${ }^{13}$, mientras que Chile no acepta en su legalidad ninguna causal de aborto ${ }^{14}$. Adicionalmente, Cuba tiene tasas de mortalidad infantil menores que las de Chile, lo cual no se explica completamente por diferencias en cobertura o en tipo de sistemas de salud.

El objetivo de este estudio es poner a prueba nuestra hipótesis que la menor mortalidad infantil en Cuba respecto a Chile, se debe en parte a la existencia de aborto eugenésico en Cuba, comparando entre estos países el efecto de las muertes infantiles por malformaciones congénitas y anomalías cromosómicas, sobre la tasa de mortalidad infantil.

\section{Materiales y Método}

Análisis poblacional comparativo de la mortalidad infantil, con especial énfasis en las muertes por malformaciones congénitas (MFC) y anomalías cromosómicas (AC) en el primer año de vida, ocurridas en Cuba y Chile en el año 2008. La información biodemográfica es obtenida de los anuarios de Estadísticas de Salud de $\mathrm{Cuba}^{15}$ y de Estadísticas Vitales de Chile ${ }^{16}$, correspondientes al año en análisis. Las causas de muerte relacionadas con MFC/AC se presentan según la 10a revisión de la Clasificación Internacional de Enfermedades, Traumatismos y Causas de Defunción (CIE-10) ${ }^{17}$, contenidas entre los códigos Q00-99, rotulados como "Muertes por malformaciones congénitas, deformidades y anomalías cromosómicas". Las variables analizadas son el número de muertes infantiles por malformaciones del sistema nervioso (Q00-07), circulatorio (Q20-28), respiratorio (Q30-34), digestivo (Q39-45), urinario (Q6064), osteomuscular (Q65-79) y las por anomalías cromosómicas (Q90-99). Las tasas de mortalidad infantil, neonatal precoz y tardía, y posneonatal, por MFC/AC y el resto de las causas, se expresan por mil nacidos vivos (nv). La comparación entre las causas de muertes se efectúa mediante la prueba de chi-cuadrado $\left(\chi^{2}\right)$ con corrección de Yates. El análisis de riesgo de muerte se realiza según Odds Ratio (OR) con intervalo de confianza del 95\% (IC 95\%). Se consideró diferencia significativa un $\mathrm{p}<0,05$.

\section{Resultados}

En la Tabla 1 se presenta el número de nacidos vivos, el total de muertes infantiles, el total de muertes infantiles por MFC/AC con sus respectivas tasas de mortalidad infantil por causas. Se observa que el porcentaje de muertes infantiles atribuibles a MFC/AC es significativamente mayor en Chile $(33,8 \% ; 658 / 1.948)$ que en Cuba $(19,2 \%$; $111 / 579)(\mathrm{p}<0,001)$. Además, como se observa en la Tabla 2, en Chile hubo un significativo mayor riesgo de mortalidad infantil, mortalidad infantil por malformaciones congénitas y mortalidad infantil por el resto de las causas.

En Chile, el 58,4\% (384/658) de las muertes por MFC/AC ocurren en el período neonatal precoz, el 13,5\% (89/658) en el neonatal tardío y el $28,1 \%(185 / 658)$ en el posneonatal; mientras que en Cuba estas muertes ocurren en $28,8 \%$ (32/111), el $24,3 \%(27 / 111)$ y el $46,9 \%$ (52/111), respectivamente. El riesgo de muerte infantil por malformaciones MFC/AC, en los tres períodos del primer año de vida es significativamente mayor en Chile (Tabla 3).

El análisis de riesgo de las muertes infantiles por MFC por sistemas y AC, se presentan en la Tabla 4. Hubo un riesgo significativamente ma- 
Aborto eugenésico y mortalidad infantil - E. Donoso et al

Tabla 1. Tasa de mortalidad infantil y por malformaciones congénitas/anomalías cromosómicas. Cuba y Chile, 2008

\begin{tabular}{|lccccc|}
\hline País & Nacidos vivos & $\begin{array}{c}\text { Muertes < } \mathbf{1} \text { año } \\
\text { (n) }\end{array}$ & TMI & $\begin{array}{c}\text { Muertes < } \mathbf{1} \text { año } \\
\text { MFC/AC (n) }\end{array}$ & TMI MFC/AC \\
\hline Cuba & 122.569 & 579 & 4,72 & 111 & 0,91 \\
Chile & 248.366 & 1.948 & 7,84 & 658 & 2,65 \\
\hline
\end{tabular}

TMI: tasa de mortalidad infantil (x 1.000 nv). MFC/AC: malformaciones congénitas/anomalías cromosómicas, incluidas bajo los códigos Q00-99 (CIE 10).

Tabla 2. Mortalidad infantil, por malformaciones congénitas/anomalías cromosómicas y el resto de las causas. Cuba y Chile, 2008

\begin{tabular}{|lcccc|}
\hline & TMI Chile & TMI Cuba & OR (95\% CI) & Valor p \\
\hline Mortalidad infantil & $1.948(7,84)$ & $579(4,72)$ & $1,67(1,52-1,83)$ & $<0,001$ \\
Mortalidad infantil por MFC/AC & $658(2,65)$ & $111(0,91)$ & $2,93(2,39-3,60)$ & $<0,001$ \\
\hline Mortalidad infantil por el resto de las causas & $1.290(5,19)$ & $468(3,82)$ & $1,36(1,22-1,52)$ & $<0,001$ \\
\hline
\end{tabular}

TMI: tasa de mortalidad infantil (x 1.000 nv). MFC/AC: malformaciones congénitas/anomalías cromosómicas, incluidas bajo los códigos Q00-99 (CIE 10).

Tabla 3. Mortalidad neonatal precoz, neonatal tardía y posneonatal por malformaciones congénitas y anomalías cromosómicas. Cuba y Chile, 2008

\begin{tabular}{|lcccc|}
\hline $\begin{array}{l}\text { Muertes }<\mathbf{1} \text { año } \\
\text { MFC/AC }\end{array}$ & $\begin{array}{c}\text { Chile } \\
\mathbf{n}(\mathbf{T M I})\end{array}$ & $\begin{array}{c}\text { Cuba } \\
\mathbf{n}(\mathbf{T M I})\end{array}$ & OR (95\% CI) & Valor p \\
$\begin{array}{l}\text { Neonatal precoz } \\
(<7 \text { días })\end{array}$ & $384(1,55)$ & $32(0,26)$ & $5,93(4,08-8,66)$ & $<0,0001$ \\
$\begin{array}{l}\text { Neonatal tardía } \\
(7-27 \text { días })\end{array}$ & $89(0,36)$ & $27(0,22)$ & $1,63(1,04-2,56)$ & $<0,03$ \\
$\begin{array}{l}\text { Posneonatal } \\
(28 \text { días }-11 \text { meses })\end{array}$ & $185(0,74)$ & $52(0,42)$ & $1,76(1,28-2,42)$ & $<0,001$ \\
\hline
\end{tabular}

TMI: tasa de mortalidad infantil (x 1.000 nv). MFC/AC: malformaciones congénitas/anomalías cromosómicas, incluidas bajo los códigos Q00-99 (CIE 10).

Tabla 4. Riesgo de mortalidad infantil por malformaciones congénitas de los sistemas y anomalías cromosómicas. Cuba y Chile, 2008

\begin{tabular}{|llrrrrrr|}
\hline & \multicolumn{2}{c}{ Cuba } & \multicolumn{2}{c}{ Chile } & & \\
CIE 10 & Sistemas & $\mathbf{n}$ & TMI & $\mathbf{n}$ & TMI & OR (IC95\%) & Valor $\mathbf{p}$ \\
Q00-07 & Nervioso & 4 & 0,03 & 107 & 0,43 & $13,21(4,70-42,04)$ & $<0,001$ \\
Q20-28 & Cardiovascular & 57 & 0,47 & 170 & 0,68 & $1,47(1,08-2,01)$ & $<0,01$ \\
Q30-34 & Respiratorio & 4 & 0,03 & 12 & 0,05 & $1,48(0,44-5,43)$ & NS \\
Q38-45 & Digestivo & 17 & 0,14 & 22 & 0,09 & $0,64(0,33-1,26)$ & NS \\
\hline Q60-64 & Urinario & 1 & 0,01 & 64 & 0,26 & $31,59(4,76-613,59)$ & $<0,001$ \\
Q65-79 & Osteomuscular & 11 & 0,09 & 88 & 0,35 & $3,95(2,05-7,81)$ & $<0,0001$ \\
\hline Q90-99 & Cromosómicas & 7 & 0,06 & 130 & 0,52 & $9,17(4,15-21,38)$ & $<0,001$ \\
\hline
\end{tabular}

TMI: tasa de mortalidad infantil (x 1.000 nv). NS: diferencia no significativa. 
yor en Chile de muertes infantiles dependientes de malformaciones del sistema cardiovascular, osteomuscular, nervioso, urinario y por anomalías cromosómicas. No hubo diferencias significativas en el riesgo de muertes infantiles dependientes de malformaciones del sistema digestivo y respiratorio.

\section{Discusión}

Los resultados de esta investigación muestran que la mortalidad infantil es significativamente mayor en Chile que en Cuba (7,84\%o vs $4,72 \%$, $\mathrm{p}<0,001)$. Las muertes infantiles atribuidas a $\mathrm{MFC} / \mathrm{AC}$ es significativamente mayor en Chile que en Cuba (33,8\% vs 19,2\%, p < 0,001), del mismo modo, la tasa de mortalidad infantil atribuida a MFC/AC es significativamente mayor en nuestro país $(2,65 \%$ ov $0,91 \%$, $\mathrm{p}<0,001)$. El análisis de mortalidad en los tres períodos del primer año de vida, también Cuba muestra mejores indicadores que Chile. Es interesante destacar que la tasa de mortalidad infantil calculada excluyendo las MFC/ AC persiste mayor en Chile (5,19\%o vs 3,82 \%o, $\mathrm{p}<0,001)$.

La mayor mortalidad infantil por MFC/AC existente en Chile, explica parcialmente la diferencia en la mortalidad infantil de ambos países, ya que excluidas las por MFC/AC, la diferencia persiste. Esto apoya nuestra hipótesis que el aborto eugenésico, permitido en Cuba y penalizado en Chile, explica parcialmente, la mayor tasa de mortalidad infantil en Chile. En el período neonatal precoz se registra la mayor diferencia en mortalidad asociada a MFC/AC [OR 5,93 (IC95\% 4,08-8,66)]; esto apoya adicionalmente nuestra hipótesis, pues la gran mayoría de los recién nacidos con MFC fallece durante la primera semana de vida ${ }^{8,10,11}$.

En Cuba, en 1965, el gobierno de la Revolución, enmendó el Código de Defensa Social, sin cambiar el artículo 433 sobre el aborto, definiendo aborto ilegal como el practicado por personal no médico, sin el consentimiento de la mujer, con fines de lucro, fuera del hospital y en violación de cualquier otra norma establecida por el Ministerio de Salud ${ }^{18-20}$. La legislación cubana coincide con la definición de salud integral de la OMS, establecida en su Constitución desde 1946 "como un estado de completo bienestar fisico, mental y social", y no solamente la ausencia de afecciones o enfermeda- des ${ }^{21}$, permitiendo a los médicos cubanos actuar con flexibilidad en lo relacionado a la inducción de aborto $^{19}$. Por el contrario, en 1989 el gobierno Militar Chileno modificó el Código Sanitario, con el artículo 119 que establece: "No podrá ejecutarse ninguna acción cuyo fin sea provocar un aborto" 14 . Por sobre esta normativa, la legislación chilena establece en el artículo 19 de su Constitución Política que: "La ley protege la vida del que está por nacer"22.

Estas contrapuestas visiones sobre el embrión y feto, establecen en Cuba y Chile diferentes estrategias para el control de las malformaciones congénitas y las anomalías cromosómicas detectadas en el control prenatal. La legislación cubana, privilegia la salud física y psicológica de la mujer, y la hace proclive a la interrupción de la gestación, mientras que la chilena privilegia al embrión por ser considerado persona desde el momento de la concepción, y establece políticas de prevención y tratamiento postnatal.

Chile desde el 2000, fortifica las harinas con ácido fólico ${ }^{23}$, para disminuir el riesgo de defectos del tubo neural como la anencefalia y el mielomeningocele. Esta medida permitió una reducción global de $43 \%$ de las malformaciones del tubo neural en la evaluación de los primeros 2 años, demostrando ser una intervención costo-efectiva ${ }^{24,25}$. Otra medida implementada en agosto de 2002 por el Estado de Chile, es el Régimen de Garantías en Salud, instrumento de regulación sanitaria que considera el Acceso Universal y Garantías Explícitas (AUGE) de la atención y tratamiento de enfermedades de alto costo y prevalencia. Esta ley considera el tratamiento de malformaciones como las cardiopatías congénitas operables en menores de 15 años, las disrrafias espinales y las fisuras labio-palatinas ${ }^{26}$. La evaluación preliminar de experiencias nacionales señala un impacto en la reducción de las muertes neonatales por cardiopatías congénitas ${ }^{27}$.

Cuba en la tercera etapa de la implementación del Programa Nacional de Reducción de la Mortalidad Infantil (1980-1989), consideró como estrategias la incorporación prenatal de nuevas tecnologías (alfafetoproteína y ultrasonido diagnóstico) para garantizar un mejor diagnóstico de los recién nacidos, como también el desarrollo de la cirugía cardiovascular para la corrección precoz de cardiopatías congénitas complejas ${ }^{28}$.

Cuba y Chile tienen una alta cobertura de personal capacitado para realizar el control prenatal, 
siendo de $100 \%$ en la población cubana y de $96 \%$ para la población obstétrica chilena ${ }^{29}$; en ambos países se considera el ultrasonido diagnóstico como parte del control habitual del embarazo de bajo riesgo.

La ultrasonografía es el procedimiento diagnóstico más importante para la detección de anomalías estructurales del feto ${ }^{30}$, como también para la identificación de indicadores de aneuploidías ${ }^{31}$. La introducción de esta metodología en el control prenatal, permite que la identificación de una anomalía fetal estructural o genética, pueda derivar a la terminación del embarazo según la legalidad vigente del país.

Una experiencia cubana prospectiva mostró que de 128 gestantes con fetos con MFC o enfermedad genética, $108(84,4 \%)$ solicitaron el aborto, siendo las malformaciones cardíacas las más frecuentes (29,7\%), seguida por los defectos del tubo neural $(18 \%)$ y las renales $(14,8 \%)$; hubo 99\% de confirmación anatomopatológico del diagnóstico prenatal ${ }^{32}$. Otro estudio cubano mostró que en un período de 6 años se diagnosticaron 499 malformaciones de las cuales 102 (20,5\%) se interrumpieron por cardiopatía, asociadas o no a otras malformaciones mayores/menores o cromosomopatías $^{33}$. Otro estudio cubano sobre la eficacia de la ultrasonografía efectuada entre las 22-24 semanas, en el diagnóstico de malformaciones mayores, mostró una sensibilidad diagnóstica global de $74,4 \%$ y una especificidad de $97,3 \%$, con un índice Kappa de 0,66 de concordancia con las necropsias ${ }^{34}$.

La experiencia chilena sobre la capacidad diagnóstica de la ecografía entre las 18-28 semanas en población obstétrica de bajo riesgo, mostró una sensibilidad general de $50 \%$ en el diagnóstico de MFC, para las genitourinarias 78,9\%, neurológicas $76,8 \%$, músculoesqueléticas $56,6 \%$, digestivas $55,2 \%$, cardiovasculares $36,2 \%$, síndromes malformativos $44 \%$ y para trisomías $49 \%{ }^{35}$. Otro estudio chileno efectuado en una población de 1.167 fetos de bajo riesgo, la ultrasonografía de rutina entre las 22-26 semanas mostró una sensibilidad de 51,3\% para el diagnóstico de malformaciones congénitas, con una cobertura de $93,7 \%$ de la población obstétrica ${ }^{36}$.

Es importante destacar que Chile y Cuba tienen un riesgo diferente de presentar hijos con malformaciones congénitas y anomalías cromosómicas, debido al envejecimiento de la población de mujeres chilenas embarazadas y en edad fértil. La tasa específica de fecundidad por edad en Cuba de mujeres de 40-44 años (4,2 nacimientos/1.000 mujeres $40-44$ años) y de 45 49 años $(0,2 \text { nacimientos/ } 1.000 \text { mujeres } 45-49)^{15}$, es muy inferior a lo que acontece en Chile con 13,3 nacimientos/1.000 mujeres $40-44$ años y 0,7 nacimientos/ 1.000 mujeres $45-49$ años, respectivamente $^{16}$. En Chile, la tendencia de los nacimientos en mujeres de 40 o más años es significativamente ascendente y con un mayor riesgo de mortalidad infantil comparadas con mujeres de 20-34 años (OR 1,60; 95\% IC 1,51-1,70) $)^{37}$. Lo anterior se explica por el envejecimiento de la mujer chilena en edad fértil ${ }^{38}$, que sumado a las mayores expectativas laborales y educacionales, hace que ellas posterguen el embarazo a edades mayores ${ }^{39}$.

Considerando las similitudes entre Chile y Cuba, con respecto a la cobertura total de la atención prenatal, el uso rutinario del ultrasonido diagnóstico en embarazos de alto y bajo riesgo, y la adecuada tasa de detección de anomalías fetales, es posible postular que la legalización del aborto eugenésico en Chile podría reducir la mortalidad infantil, por evitar el nacimiento de fetos no viables, cuyo destino es morir en el período neonatal o dentro del primer año de vida.

Desconocemos que exista una legislación sobre aborto eugenésico que tenga como fin la reducción de la mortalidad infantil; si así fuese consideramos esa alternativa como éticamente incorrecta. La reducción de la tasa de mortalidad infantil, si bien es un objetivo sanitario mundial ${ }^{40}$, debe ser el reflejo de políticas públicas de mejoría integral de la salud del niño, independiente de su condición estructural o genética.

Para Cuba y Chile, las tasas de mortalidad infantil en 2008, han sido las más bajas para ambos países en su historia demográfica y sanitaria, con una reducción del indicador respecto al año 2000, de $16,1 \%$ para Chile y de $34,7 \%$ para Cuba ${ }^{15,16}$. Los datos presentados corresponden a un año, diferencias que podrían no repetirse en otros. Para descartar esta posibilidad, analizamos de modo similar, las estadísticas vitales de Chile y Cuba de los años 2006 y 2007, observando similares diferencias, y siempre a expensas del aumento de la mortalidad infantil asociada a MFC/AC.

Es justo enfatizar que Cuba mantiene una significativa menor tasa de mortalidad infantil que Chile en el análisis de las causas, excluidas 
las por MFC/AC (5,19\%o vs 3,82\%o, p < 0,001). Desconocemos la razón de esta diferencia, pero podemos aventurar que es el reflejo de mejores políticas públicas en salud infantil en Cuba. Una reflexión mayor sobre este tema se aleja de los objetivos de este estudio.

\section{Conclusión}

El análisis de nuestros datos apoya la hipótesis de que el diagnóstico prenatal de anomalías congénitas puede explicar parcialmente las menores tasas de mortalidad infantil de Cuba en comparación con Chile, mediante el aborto eugenésico. Así, diferentes conceptos sobre salud materna y fetal podrían influir en un sensible indicador demográfico, como es la muerte del niño en el primer año de vida.

\section{Referencias}

1. UNICEF. The state of the world's children 2008. Child survival. December 2007. Disponible en: http://www. unicef.org/sowc08/docs/sowc08.pdf. Consultado el 17 de mayo de 2009.

2. PAHO. Health situation in the Americas: basic indicator 2008. Disponible en: www.paho.org/english/dd/ais/ coredata.htm. Consultado el 17 de mayo de 2009.

3. Kaempffer AM, Medina E. [Analysis of infant mortality and associated factors. Chile 1998]. Rev Chil Pediatr 2000; 71: 405-12.

4. Riverón Corteguera R, Azcuy Henríquez P. Mortalidad infantil en Cuba 1959-1999. Rev Cubana Pediatr 2001; 73: 143-57.

5. Ewigman BG, Crane JP, Firgoletto FD, Lefevre ML, Bain $\mathrm{RP}, \mathrm{McNellis} \mathrm{D}$, and the Radius study group. Effect of prenatal ultrasound screening on perinatal outcome. $\mathrm{N}$ Engl J Med 1993; 329: 821-7.

6. Levi S, Schaaps JP, De Havay P, Coulon R, Defoort P. End-results of routine ultrasound screening for congenital anomalies: the Belgian Multicentric Study 1984-92. Ultrasound Obstet Gynecol 1995; 5: 366-71.

7. Grandjean H, Larroque D, Levi S. The performance of routine ultrasonographic screening of pregnancies in the Eurofetus study. Am J Obstet Gynecol 1999; 181: 446-54.

8. Wen SW, Liu S, Joseph KS, Rouleau J, Allen A. Patterns of infant mortality caused by major congenital anomalies. Teratology 2000; 61: 342-6.

9. Liu S, Joseph KS, Kramer MS, Allen AC, Sauve R, Rusen
ID, Wen SW; Fetal and Infant Health Study Group of the Canadian Perinatal Surveillance System. Relationship of prenatal diagnosis and pregnancy termination to overall infant mortality in Canada. JAMA 2002; 287: 1561-7.

10. Liu S, Joseph KS, Wen SW. Trends in fetal and infant deaths caused by congenital anomalies. Semin Perinatol 2002; 26: 268-76.

11. Forrester MB, Merz RD. First-year mortality rates for selected birth defects, Hawaii, 1986-1999. Am J Med Genet A 2003; 119A: 311-8.

12. Mangione R, Fries N, Godard P, Fontanges M, Haddad G, Mirlesse V; College Français D’Echographie Foetal (CFEF). [Outcome of fetuses with malformations discovered before 14 weeks. Where the discovery is revealed by echography during the first trimester, is it responsible for the voluntary termination of the pregnancy? Comparison before and after July 2001]. J Gynecol Obstet Biol Reprod (Paris) 2008; 37: 154-62.

13. Código Penal de Cuba. Título VIII. Delitos contra la vida y la integridad corporal. Capítulo VI. Aborto ilícito: artículos 267 al 271. Ley No 62 del 29 de diciembre de 1987.

14. Código Sanitario, República de Chile. Libro V. Del ejercicio de la medicina y profesiones afines. Artículo 119. Ley No 18.826 del 15 de septiembre de 1989.

15. Dirección Nacional de Estadísticas de Cuba. Anuario estadístico de salud, Cuba 2008. Disponible en: http:// www.sld.cu/servicios/estadisticas/. Consultado el 16 de mayo de 2011.

16. Instituto Nacional de Estadísticas de Chile. Anuario de Estadísticas Vitales, Chile 2008.

17. WHO. International Statistical Classification of Diseases and Related Health Problems. Tenth Revision. Geneva, World Health Organization, 1992.

18. Alvarez-Lajonchere C. Commentary on abortion law and practice in Cuba. Int J Gynecol Obstet 1989; Suppl 3: 93-5.

19. Castillo-Vargas R, González-Cárdenas L, Erice-Candelario A, Castillo-Duranza R. Bioethical perspectives: Abortion, de jure and de facto situation. Arch Med Fam 2002; 4: 75-6.

20. Castillo-Vargas R, González-Cárdenas L. Abortion: the essential problem of bioethics. Arch Med Fam 2004; 6: 34-5.

21. WHO. Constitución de la Organización mundial de la Salud. Disponible en: http://www.who.int/governance/ eb/who_constitution_sp.pdf. Consultado el 15 de mayo de 2011.

22. Constitución Política de la República de Chile. Decreto Supremo No 1.150 de 1980. Disponible en: http://www. paginaschile.cl/biblioteca_juridica/constitucion.htm. 
Consultado el 3 de junio de 2011.

23. Hertrampf E, Cortés F, Erickson JD, Cayazzo M, Freire W, Bailey LB, Howson C, Kauwell GP, Pfeiffer C. Consumption of folic acid-fortified bread improves folate status in women of reproductive age in Chile. J Nutr 2003; 133: 3166-9.

24. Llanos A, Hertrampf E, Cortés F, Pardo A, Grosse SD, Uauy R. Cost-effectiveness of a folic acid fortification program in Chile. Health Policy 2007; 83: 295-303.

25. Hertrampf E, Cortés F. National food-fortification program with folic acid in Chile. Food Nutr Bull 2008; 29 (2 Suppl): S231-7.

26. República de Chile. Ley 19.966. Garantías explícitas en salud. Disponible en: http://www.redsalud.gov.cl/ archivos/guiasges/GES_2007_final.pdf. Consultado el 7 de junio de 2011.

27. Concha F, Pastén N, Espinoza V, López F. Impacto de la implementación del Plan AUGE en la detección antenatal de cardiopatías congénitas. Rev Chil Obstet Ginecol 2008; 73: 163-72.

28. Riverón Corteguera R. Estrategias para reducir la mortalidad infantil, Cuba 1959-1999. Rev Cubana Pediatr 2000; 72: 147-64.

29. PAHO. Health Information and Analysis. Health situation in the Americas: Basic Indicators 2008. Washington, D.C., 2008.

30. Levi S. Mass screening for fetal malformation: the Eurofetus Study. Ultrasound Obstet Gynecol 2003; 22: 555-8.

31. Cicero S, Sacchini C, Rembouskos G, Nicolaides KH. Sonographic markers of fetal aneuploidy--a review. Placenta 2003;24 Suppl B:S88-98.

32. Piloto Morejón M, Sanabria Chocontá MI, Menéndez García R. Diagnóstico prenatal y atención de las malformaciones congénitas y otras enfermedades genéticas. Rev Cubana Obstet Ginecol 2001; 27: 233-40.

33. Martínez González RL, Valladares Hernández M, Pé- rez Martínez C. Cardiopatía congénita. Diagnóstico e interrupciones en nuestra Provincia, 1994-1999. Rev Cubana Obstet Ginecol 2001; 27: 146-51.

34. González Jiménez G, Gómez Vatue R, González Iglesias Y. Evaluación de la eficacia diagnóstica por ultrasonografía en malformaciones congénitas mayores. Rev $\mathrm{Cu}$ bana Obstet Ginecol [on line] 2002; 28: 0-0. Disponible en: http://scielo.sld.cu/pdf/gin/v28n3/gin01302.pdf. Consultado el 3 de diciembre de 2010.

35. González R, Carstens M, Gómez R, Medina L, Gutiérrez J, Arriagada P, Nien Ch-J, Álvarez MJ, González C, Nazal L, Rojas I. Anomalías congénitas en población de bajo riesgo: screening con ultrasonido prenatal en un sistema público de salud. Rev Chil Obstet Ginecol 1999; 64: 247 55.

36. González R, Dezerega V, Vásquez R. Contribución a la ecografía rutinaria en el período 22 a 26 semanas al diagnóstico de anomalías congénitas. Experiencia de 2 años, en 1162 ecografías. Rev Chil Obstet Ginecol 2005; 70: 289-95.

37. Donoso E, Becker J, Villarroel L. Evolución de la natalidad y del riesgo reproducido en mujeres de 40 o más años en la década de los 90. Rev Chil Obstet Ginecol 2002; 67: 139-42.

38. Donoso E, Carvajal J, Domínguez MA. [Fecundity reduction and aging in fertile women population in the period 1990-2004 in Chile]. Rev Med Chile 2009; 137: 766-73.

39. Larrañaga O. Participación laboral de la mujer en Chile: 1958-2003. Disponible en: http://economia.uahurtado. cl/pdf/seminarios/larranaga.pdf. Consultado el 5 de diciembre de 2010.

40. United Nations. Millennium Development Goals. Goal 4: reduce child mortality. Disponible en: http://www. un.org/millenniumgoals/childhealth.shtml. Consultado el 7 de febrero de 2011. 